da die Angabe in Watt sich auf den ganzen Empfindlichkeitsbereich bezieht und nicht auf eine Wellenlänge. Unabhängig davon sieht man aber,

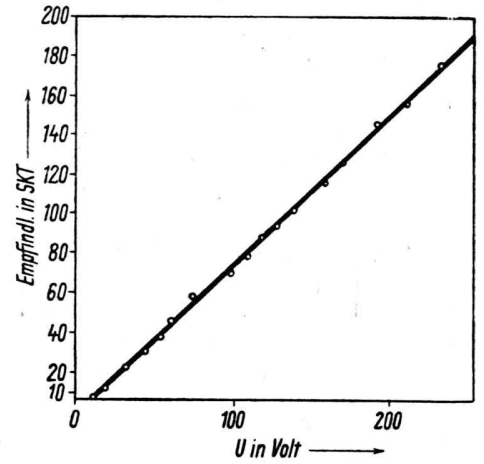

Abb. 4. Abhängigkeit des Lichtstromes von der angelegten Spannung.

daß die CdS-Zelle in dieser Form an Empfindlichkeit dem menschlichen Auge am nächsten kommt. Es sei noch erwähnt, daß die Photozelle im April bei Tageslichtbeleuchtung einen Strom von $80 \mathrm{~mA}$ lieferte.

Abb. 3 gibt den spektralen Empfindlichkeitsverlauf zweier Zellen wieder. Durch besonderen Aufbau war es nach Abb. 3 (I) möglich, die Empfindlichkeit nach der langwelligen Seite des Spektrums hinauszuschieben.

Abb. 4 zeigt die Abhängigkeit des Photostromes von der angelegten Spannung. Wie man sieht, besteht in dem angegebenen Intervall ein linearer Zusammenhang zwischen Spannung und Strom.

Abb. 5 gibt den Zusammenhang von Temperatur und Leitfähigkeit. Bis $80^{\circ}$ findet kaum eine Leitfähigkeitszunahme statt, und erst bei $200^{\circ}$ steigt die Kurve steil an.

Die für die Sulfidphosphore charakteristische Tilgung konnte an den CdS-Schichten ebenfalls beobachtet werden. Wurde eine belichtete Zelle zusätzlich mit ultraroten Wellen bestrahlt, so ging der Galvanometerausschlag etwa auf die Hälfte zurück. Ebenso trat bei Belichtung eine selbständige EMK auf. Eine weitere Entwicklung zum CdS-Photoelement halte ich durchaus für möglich.

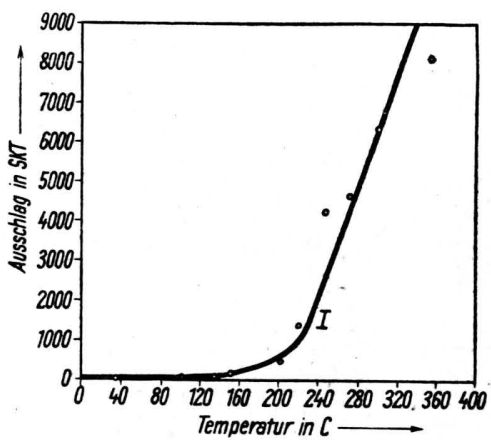

Abb. 5. Abhängigkeit der Leitfähigkeit einer CdSSchicht von der Temperatur bei der Abkühlung.

\title{
Die Aufnahme quantitativer Absorptionsspektren fester Stoffe in Reflexion
}

\author{
Von Gustav Kortüm und Maria Kortüm-SeIler \\ Aus dem Physikalísch-chemischen Institut der Universität Tübingen \\ (Z. Naturforschg. 2a, 652-657 [1947]; eingegangen am 13. August 1947)
}

\begin{abstract}
Die Bedingungen, unter denen sich aus der Intensitätsverteilung der an matten Oberflächen diffus und regulär reflektierten Strahlung das Absorptionsspektrum des reflektierenden Stoffes ermitteln läßt, werden diskutiert. Es wird eine Methode ausgearbeitet, nach der die quantitativen Absorptionsspektren fein pulverisierter Stoffe in Reflexion reproduzierbar mit einer Fehlergrenze von $\pm 5 \%$ der Extinktion photographisch aufgenommen werden können. Die Methode wird durch Messungen an $\mathrm{K}_{2} \mathrm{CrO}_{4}$ unter Variation der äußeren Bedingungen (Korngröße, Vergleichsstandard, Einfalls- und Austrittswinkel der Strahlung) geprüft. Die starke Beeinflußbarkeit der längstwelligen $\mathrm{CrO}_{4}^{2-}$ Bande durch die Korngröße des Kristallpulvers wird auf die Überlagerung von Gitterund Radikalschwingungen bei dieser Elektronenanregung zurückgeführt.
\end{abstract}

$\ddot{U}$ ber die Absorptionsspektren fester Stoffe liegen im Vergleich zu den zahllosen Untersuchungen an Lösungen und Gasen nur wenig Messungen vor. Ihre Aufnahme scheitert gewöhnlich an der Schwierigkeit, genügend dünne und definierte Schichten herzustellen. Die dafür benutzten Methoden (Züchtung geeigneter Kristallblättchen, Aufdampfen auf Quarzplatten im Vakuum, Schmelzen und Erstarrenlassen zwischen zwei Quarzplatten, Verreiben des Kristallpulvers 
auf einer mattierten Quarzplatte usw.) sind häufig nicht anwendbar und liefern außerdem Schichtdicken, die ungleichmäßig und schwer auszumessen sind und in der Regel stark streuen, so daß die Messungen nicht quantitativ ausgewertet werden können.

Es ist deshalb mehrfach versucht worden ${ }^{1}$, eine allgemeiner verwendbare Methode auszuarbeiten, die auf der Messung der Reflexion an Kristallpulvern beruht, ohne daß diese Versuche bisher zu befriedigenden Erfolgen geführt haben.

\section{Methodische Grundlagen}

Grundsätzlich ist zwischen zwei Anteilen der Reflexion zu unterscheiden, der ,regulären" Reflexion an spiegelnden Flächen und der „diffusen“" Reflexion, bei der das Licht in tiefere Schichten eindringt und durch mehrfache Streuung an die Oberfläche zurückgelangt. Wir betrachten zunächst den einfachsten Fall einer vollkommen diffusen Reflexion paralleler monochromatischer Strahlung an einer nichtabsorbierenden matten Oberfläche. Die Bestrahlungsstärke $E$ ist definiert als die auf die Flächeneinheit einfallende Strahlungsleistung (Lichtstrom), gemessen z. B. in Watt $/ \mathrm{cm}^{2}$

$$
E=\frac{d \Phi}{d f}
$$

Die Bestrahlungsstärke hängt vom Cosinus des Einfallswinkels $\vartheta \mathrm{ab}$ :

$$
E=E_{0} \cos \vartheta
$$

wenn $E_{0}$ die Bestrahlungsstärke bei senkrechtem Einfall bedeutet (vergl. Abb.1). Aus den beiden Gleichungen folgt

$$
d \Phi_{e}=E_{0} \cos \vartheta d f,
$$

und für die gesamte, von der Fläche $f$ aufgenommene Strahlungsleistung

$$
\Phi_{e}=E_{0} \cos \vartheta f
$$

1 Vergl. C. F. Go o d e ve, Trans. Faraday Soc. 33, 340 [1937] ; L. A m y, Rev. Opt. théor. instrument. 16, 81 [1937]; Ch. Sannie, L. A m y u. J. M. Sarraf, Rev. Opt. théor. instrument. 16, 86 [1937]; T. G u i l $\mathrm{mart} u$. R. Fre y m a n n, Rev. Opt. théor. instrument. 17, 199 [1938]; T. G u i l ma r t, Bull. Soc. Chimique de France (V) 5, 1209 [1938]; M. B i lly u. A. B e r t o n , C. R. hebd. Séances Acad. Sci. 206, 1631 [1938] ; L. A m y, C. R. hebd. Séances Acad. Sci. 207, 329 [1938].
Die Ausstrahlung der aufgenommenen Leistung erfolgt bei vollkommen diffuser Reflexion nach allen Richtungen der Halbkugel. Bezeichnet man

Abb. 1.

Zur Ableitung des Cosinus-Gesetzes.

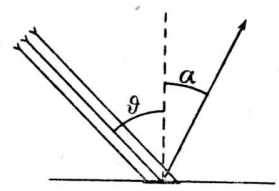

die in Richtung $\alpha$ in den Einheitsraumwinkel ausgestrahlte Leistung

$$
\frac{d \Phi}{d \omega}=J_{a}
$$

als Strahlungsstärke (Lichtstärke), gemessen z. B. in Hefnerkerzen, so gilt nach dem von $\mathrm{L}$ a m be r t empirisch gefundenen Gesetz für die räumliche Verteilung der Leistung (vergl. Abb. 1)

Die Konstante

$$
J_{a}=B f \cos a .
$$

$$
B=\frac{J_{a}}{f \cos a}
$$

wird als Strahlungsdichte (Leuchtdichte, Flächenhelligkeit) bezeichnet und z. B. in Hefnerkerzen $/ \mathrm{cm}^{2}=$ Stilb gemessen.

Aus (5) und (6) folgt.

$$
d \Phi_{a}=B f \cos \alpha d \omega .
$$

Ersetzt man den Raumwinkel $d \omega$ durch das Flächenelement auf der Einheitskugel

$$
d \omega=\sin \alpha d \alpha d \varphi
$$

und integriert über die gesamte Halbkugel, so erhält man für die insgesamt abgegebene Strahlungsleistung

$$
\begin{aligned}
\Phi_{a} & =B f \int_{0}^{\pi / 2} \int_{0}^{\pi / 2} \cos \alpha \sin \alpha d a d \varphi \\
& =2 \pi B f \int_{0}^{\pi / 2} \cos a \sin \alpha d a=\pi B f=\frac{\pi J_{a}}{\cos \alpha} .
\end{aligned}
$$

Da nun für nichtabsorbierende Stoffe aufgenommene und durch diffuse Reflexion abgegebene Strahlungsleistung gleich sein müssen, folgt aus (4) und (10) $\Phi_{e}=\Phi_{a}$ oder

$$
J_{a}=\frac{E_{0} f}{\pi} \cdot \cos \vartheta \cos \alpha=k \cos \vartheta \cos \alpha .
$$


Die Stärke der reflektierten Strahlung ist dem Cosinus des Einfalls- und des Austrittswinkels proportional, und der Proportionalitätsfaktor $k$ hängt ausschließlich von der primären Bestrahlungsstärke und der Größe der bestrahlten Oberfläche $a b$.

Wie die Erfahrung zeigt ${ }^{2}$, ist Gl. (11) bei matten Oberflächen wie $\mathrm{CaCO}_{3}, \mathrm{MgO}, \mathrm{BaSO}_{4}$ nur angenähert, d. h. in einem beschränkten Winkelbereich von $\vartheta$ und $\alpha$ erfüllt, es gibt also eine vollkommen diffuse Reflexion ebensowenig wie vollkommen regulär reflektierende Spiegel. Häufig findet man eine beträchtlich verstärkte Reflexion in Richtung der regulären Spiegelung, was darauf hinweist, daß die Oberfläche nicht genügend,,matt “ ist. So konnte Wright nachweisen, daß bei gepreßten Pulvern (Teilchengröße $\sim 2 \mu$ ) für konstanten Einfallswinkel थ das Cosinus-Gesetz gilt, während bei konstantem $\alpha$ die Stärke des reflektierten Lichtes dem $\cos \vartheta$ nicht proportional war. Im allgemeinen findet man Gl. (11) für kleine Werte von $\vartheta$ und $\alpha$ innerhalb der Meßgenauigkeit photometrischer Methoden erfüllt. Größere Abweichungen treten häufig dann auf, wenn Eintrittsund Austrittsebene des Lichtes nicht zusammenfallen. Alle diese Beobachtungen weisen darauf hin, daß auch bei der Reflexion an sehr feinen Pulvern der reguläre Anteil nicht immer zu vernachlässigen ist. Es hat deshalb nicht an Versuchen gefehlt, ein Reflexionsgesetz auf theoretischem Wege abzuleiten ${ }^{3}$. Man ging dabei gewöhnlich von der Vorstellung aus, daß sich die matte Oberfläche eines diffus reflektierenden Stoffes aus einer großen Zahl kleiner, regulär spiegelnder Flächen zusammensetzt, die unter allen möglichen Winkeln gegen die makroskopische Oberfläche geneigt sind. Der von diesen Spiegeln regulär reflektierten Strahlung der Stärke $J^{\prime}$ überlagert sich eine weitere der Stärke $J^{\prime \prime}$, die nach dem Eindringen in tiefere Schichten durch Zerstreuung wieder an die Oberfläche gelangt. Zur Berechnung von $J^{\prime \prime}$ wird die strenge Gïltigkeit des Cosinus-Gesetzes (11) vor-

2 Vgl. z. B. H. Wright, Ann. Physik 1, 17 [1900]; F. Jentzs ch, Ann. Physik 39, 997 [1912]: F. Henning u. W. He use, Z. Physik 10, 111 [1922]; G. P. Wor on of f u. G. J. Pokrowski, Z. Physik 20, 358 [1924] ; H. Sc h u l z, Z. Physik 31, 496 [1925].

3 Vgl. E. M. B e r r y, J. opt. Soc. America 7, 627 [1923]; G. J. P ok r o w s k i, Z. Physik 30, 66 [1924]; 35, 34, 390 [1926] ; 36, 472 [1926]; M.Le on tow it sch. Z. Physik 46, 739 [1928]; H. L i t t m a n n, Ann. Physik 38, 139 [1940]. ausgesetzt, die Größe von $J^{\prime}$ hängt nach den $\mathrm{Fr}$ e's nel schen Formeln von den Winkeln $\&$ und $\alpha$, dem Brechungsindex $n$ des Stoffes und außerdem von der wahrscheinlichsten Verteilung der Elementarspiegel über die verschiedenen Richtungen ab. Je nach den hierfür gemachten Annahmen gelangt man zu etwas verschiedenen Formeln für die gesamte Reflexionsstärke, ohne daß jedoch alle Beobachtungen durch die abgeleiteten Gleichungen erfaßt werden können.

Arbeitet man nicht mit monochromatischer, sondern mit kontinuierlicher Strahlung, und zeigt der reflektierende Stoff selektive Absorption, so wird die Stärke der reflektierten Strahlung und damit die Konstante $k$ in Gl. (11) von der Wellenlänge abhängig, und zwar gilt dies sowohl für den regulär wie für den diffus reflektierten Anteil. Da der Brechungsindex $n$ im Bereich der Absorptionsbanden infolge der anomalen Dispersion extreme Werte annimmt, ändert sich dort auch die reguläre Reflexion $\left(J^{\prime}\right)$ nach dem Fresn e l-B e e r schen Gesetz sehr stark, so daß die spektrale Intensitätsverteilung der reflektierten Strahlung von der Intensitätsverteilung der einfallenden Strah lung abweicht ${ }^{4}$. Da der größere Teil der auffallenden Strahlung in das Innere der Substanz eindringt und durch mehrfache Streuung an die Oberfläche zurückgelangt $\left(J^{\prime \prime}\right)$, wird er im Bereich der selektiven Absorption nach dem $\mathrm{L}$ a $\mathrm{m}$ ber t schen Gesetz $\log J_{0} / J=\varepsilon d$ geschwächt. Bei genügender Feinheit des Pulvers und entsprechend häufiger Streuung ist die im Mittel durchlaufene Schichtdicke $d$ von der Wellenlänge annähernd unabhängig ${ }^{5}$, so daß die spektrale Intensitätsverteilung des diffus reflektierten Anteils $\left(J^{\prime \prime}\right)$ im Vergleich zu der spektralen Intensitätsverteilung bei der diffusen Reflexion an einem nichtabsorbierenden Pulver $\left(J_{0}{ }^{\prime \prime}\right)$ das Absorptionsspektrum des zu untersuchenden Stoffes darstellt:

$$
E=\log \frac{J_{0}^{\prime \prime}}{J^{\prime \prime}}=\varepsilon \bar{d} .
$$

${ }_{4}$ Diese Abweichungen sind besonders im Ultrarot sehr stark, so daß man durch mehrfache Reflexion an spiegelnden Flächen nahezu monochromatische Strahlung herstellen kann (Reststrahlmethode).

5 Dies gilt wegen der anomalen Dispersion der Strahlung sicher nicht streng. Dả der Brechungsindex im langwelligen Teil einer Bande extrem große, im kurzwelligen extrem kleine Werte annimmt, ist die im Mittel durchlaufene Schichtdicke für lange Wellen geringer als für kurze. Das bedeutet, daß die Extinktion auf der langwelligen Seite der Bande zu klein, auf der kurzwelligen zu groß wird, so daß die Bande etwas nach kurzen Wellen verschoben sein kann. 
Je vollkommener die diffuse Reflexion ist, d. h. je besser das Cosinus-Gesetz (11) für monochromatische Strahlung gültig ist - abgesehen von der Größe der Konstante $k$ - um so besser wird das Reflexionsspektrum mit dem wahren $\mathrm{Ab}$ sorptionsspektrum übereinstimmen ${ }^{6}$. Nun hat man gerade bei absorbierenden Stoffen häufig nur eine begrenzte Gültigkeit des Cosinus-Gesetzes gefunden, was darauf hindeutet, daß man den Anteil der regulären Reflexion nicht immer vernachlässigen kann. Für die praktische Anwendbarkeit der Methode zur Ermittlung von Absorptionsspektren ist deshalb zu untersuchen, unter welchen Bedingungen dieser Anteil die Messungen nicht mehr innerhalb der Meßgenauigkeit zu fälschen vermag. Nach den oben diskutierten Erfahrungen dürfte diese Voraussetzung bei kleinen Einfalls- und Retlexionswinkeln und bei möglichst matter Oberfläche der zu untersuchendén Kristallpulver weitestgehend erfüllt sein.

\section{Experimentelle Durchführung}

Die von uns verwendete Versuchsanordnung ist in Abb. 2 schematisch dargestellt. Das zu untersuchende Pulver und der nichtabsorbierende Bezugsstandard $\left(\mathrm{BaSO}_{4}\right.$ oder $\left.\mathrm{MgO}\right)$ befanden sich in zwei in einem Metallschlitten eingelassenen Vertiefungen, so daß sie abwechselnd in den Strahlengang gebracht werden konnten. Zur Beleuchtung diente eine Xenon-Hochdrucklampe (Osram HBO 321) mit kontinuierlichem Spektrum und großer Intensität, deren Bogen vergröBert auf dem Pulver abgebildet wurde, so daß dieses gleichmäßig beleuchtet war: die beleuchtete Schicht wurde mit einer zweiten Quarzlinse (nicht ganz scharf ${ }^{7}$ ) auf dem Spektrographenspalt abgebildet. Die Reflexionsspektren beider Stoffe wurden nacheinander und mit Hilfe zweier Spaltblenden unmittelbar übereinanderliegend aufgenommen, wobei die von dem Vergleichsstandard ausgehende Strahlung durch einen Scheibe-Sektor und zusätzlich durch Raster be. kannter Extinktion meßbar geschwächt wurde. Auf den so entstandenen Doppelspektren wurden die Stellen gleicher Schwärzung mit Hilfe eines lichtelektrischen Plattenmeßapparates als Funktion der Wellenlänge bestimmt ${ }^{8}$. Zur graphischen Darstellung der Spektren w urden die aus der Sektoröffnung bekannten Extinktionen gegen die Wellenzahlen in $\mathrm{cm}^{-1}$ aufgetragen. Als Spektrograph diente der Quarzspektrograph $110 \mathrm{c}$ von Fuess, die Belichtungszeiten variierten (bei Benutzung von Agfa-Platten Spektral-gelb-

${ }^{6}$ Dabei ist zu berücksichtigen, daß man auch mit durchfallender Strahlung bei einheitlichen Kristallen definierter Schichtdicke kein "quantitatives" Absorptionsspektrum erhält, weil die Verluste durch reguläre Reflexion an den Grenzflächen nicht wie bei Lösungen und Gasen eliminiert werden können. rapid und einer Spaltbreite von $0,2 \mathrm{~mm}$ ) zwischen $30 \mathrm{sec}$ (für $E=0,6$ ) und $4 \mathrm{~min}$ (für $E=1,8$ ).

Auf die Herstellung möglichst matter Schichten wurde besondere Sorgfalt verwendet. Bei starkem Pres-

Abb. 2. Schema der Versuchsanordnung.

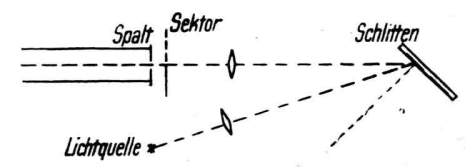

sen bilden sich stets spiegelnde Flächen. Gleichmäßig matte Oberflächen erhält man, wenn man das Pulver mit einem mattierten Glasstempel unter schwachem Druck und leichtem Drehen in die Vertiefungen einpreßt. Durch Anwärmen des Stempels läßt sich das Ankleben des Pulvers am Stempel vermeiden.

$$
\text { Meßergebnisse }
$$

Um die Brauchbarkeit und die Leistungsgrenzen der Methode zu prüfen, haben wir das Spektrum des $\mathrm{K}_{2} \mathrm{CrO}_{4}$ unter verschiedenen Bedingungen und unter Verwendung von frisch gefälltem $\mathrm{BaSO}_{4}$ als Vergleichsstandard aufgenommen.

Abb. 3 zeigt das Spektrum eines feinst pulverisierten Präparats in Abhängigkeit von Einfallsund Austrittswinkel der Strahlung. Es wurde bei feststehender Lichtquelle der die Präparate tragende Schlitten so gedreht, daß einmal der auf-

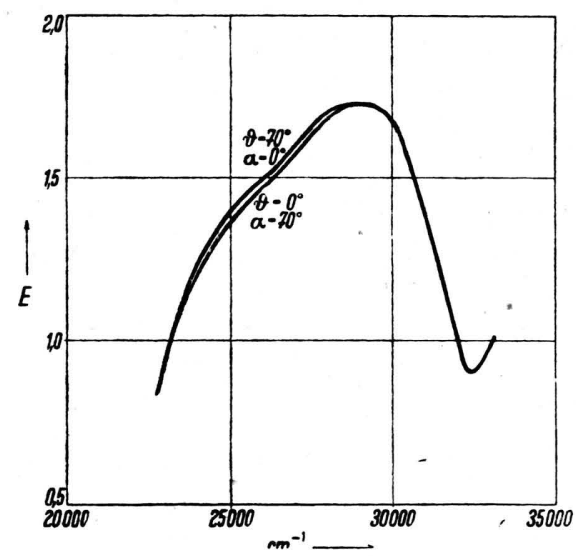

Abh. 3. $\mathrm{K}_{2} \mathrm{CrO}_{4}$-Spektrum in Abhängigkeit von Einfalls- und Austrittswinkel. I: $\vartheta=0, \alpha=70^{\circ}$; II: $\vartheta=70^{\circ} ; \alpha=0$.

fallende, einmal der reflektierte Strahl senkrecht zur Oberfläche stand. Aus der (wiederholt festgestellten) Tatsache, daß die Spektren innerhalb

7 Bei scharfer Abbildung beobachtet man Streifenbildung im Spektrum, wie schon Guilmart und F r e y m a $\mathrm{n} \mathrm{n}^{\mathbf{1}}$ bemerkt haben.

8 Vergl. G. K o r tü m, Kolorimetrie und Spektralphotometrie, Berlin 1941. 
der Meßgenauigkeit zusammenfallen, geht hervor, daß unter den gegebenen Verhältnissen für die Form der Kurve nicht die einzelnen Winkel $\vartheta$ und $\propto$, sondern nur ihre Summe maßgebend ist.

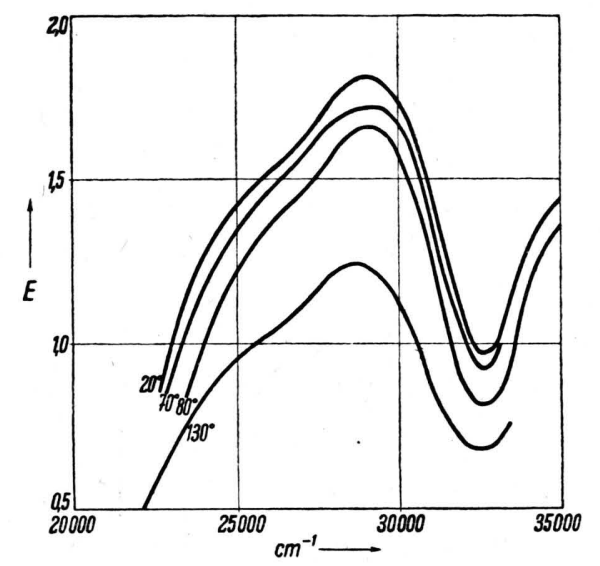

Abb. 4. $\mathrm{K}_{2} \mathrm{CrO}_{4}$-Spektrum mit $(\alpha+\vartheta)$ als Parameter.

Abb. 4 zeigt die Abhängigkeit des Spektrums von der Summe $(\alpha+\vartheta)$. Die Absorption nimmt mit zunehmendem Winkel zwischen einfallendem und austretendem Strahl ab, und zwar um so stärker, je größer dieser Winkel wird, jedoch liegt diese Abnahme bis $(\alpha+\vartheta)=45^{\circ}$ innerhalb der Fehlergrenze der Methode $( \pm 5 \%$ in $E)$, so daß man mit kleineren Winkeln reproduzierbare Spektren erhält. Die Intensitätsabnahme der Absorption besteht nicht lediglich in einer Parallelverschiebung der Kurve, sondern in einer Verflachung des ganzen Spektrums. Darin kommt der Unterschied der Winkelabhängigkeit der Reflexion an Substanz und Vergleichsstandard zum Ausdruck, der durch die verschiedenen Beiträge der regulären Reflexion bedingt ist und gerade im $\mathrm{Be}$ reich der Absorptionsbande besonders groß wird. Die Reproduzierbarkeit der Spektren bei kleinen Winkeln $(\alpha+\vartheta)$ läßt darauf schließen, daß hier der Anteil der regulären Reflexion genügend klein ist, so daß er die Messungen praktisch nicht beeinflußt. Dies wird auch dadurch bestätigt, daß für $(\alpha+\vartheta)<45^{\circ}$ das Spektrum sich nicht ändert, wenn man einen andern Vergleichsstandard ( $\mathrm{MgO}$ ) verwendet.

In Abb. 5 ist in den Kurven II bis IV die Abhängigkeit des Spektrums von der Korngröße des Kristallpulvers dargestellt. Das in Wasser gelöste * $\mathrm{K}_{2} \mathrm{CrO}_{4}$ wurde mit Alkohol ausgefällt und das feinkristallisiert ausfallende Salz verschieden lang (30 Min. bis zu mehreren Tagen) im Achat- mörser zerrieben. Die feinste Probe (Kurve IV) bestand unter dem Meßmikroskop aus Kriställchen mit Abmessungen unter 1, bis $2 \mu$. Das als Vergleichsstándard benutzte frisch gefällte $\mathrm{BaSO}_{4}$ war noch etwas feiner. Die Kurve $\mathrm{V}$ zeigt zum Vergleich das Absorptionsspektrum in wäßriger Lösung, die Kurve I das Spektrum eines kompàkten großen Kristalls, das allerdings wegen der Spiegelreflexion in seiner Intensität nicht streng reproduzierbar war.

Das Spektrum des $\mathrm{K}_{2} \mathrm{CrO}_{4}$-Kristalls zeigt, übereinstimmend mit früheren Messungen im durchfallenden Licht ${ }^{9}$, drei Bandensysteme, die verschiedenen Elektronenübergängen in der $3 d$ Schale des Cr-Atoms zugeordnet werden. Das

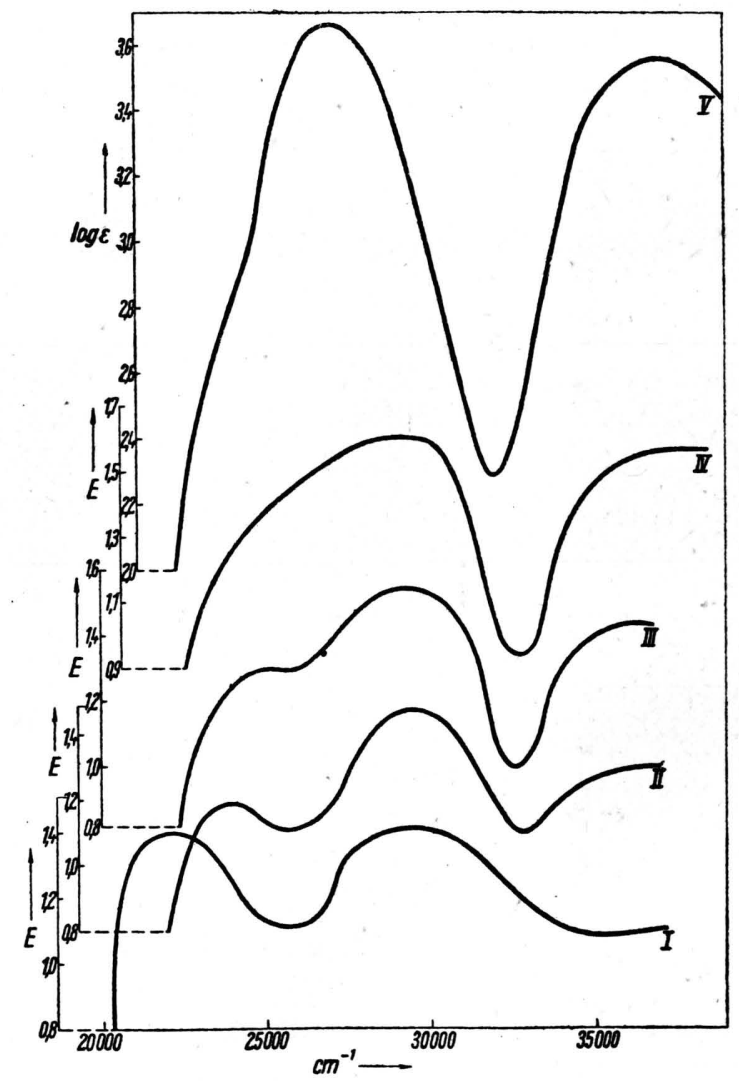

Abb. 5. $\mathrm{K}_{2} \mathrm{CrO}_{4}$-Spektrum in Abhängigkeit von der Korngröße des Kristallpulvers.

Hauptmaximum bei $29500 \mathrm{~cm}^{-1}$ ist gegenüber dem der wäßrigen Lösung um etwa $2500 \mathrm{~cm}^{-1}$, gegenüber dem von $\mathrm{Seh}$ a u man $\mathrm{n}^{9}$ in Durchsicht bei Zimmertemperatur beobachteten um rd. $2000 \mathrm{~cm}^{-1}$

${ }^{9}$ H. S ch a u mann, Z. Physik 76, 106 [1932]; J. Telt ow, Z. physik. Chem., Abt. B, 43, 198 [1939]. 
nach kurzen Wellen verschoben. Die nach der Reflexionsmethode bestimmten Maxima fallen also mit den nach der üblichen Methode gemessenen nicht zusammen, was nach den oben angestellten Überlegungen auf die Abhängigkeit der im Mittel durchlaufenen Schichtdicke von der Wellenlänge im Gebiet der anomalen Dispersion zurückzuführen sein dürfte ${ }^{10}$. Über das Maximum der längstwelligen Bande liegen keine früheren Angaben vor.

Man sieht aus den Kurven I bis IV, daß die in diffuser Reflexion aufgenommenen Kurven mit abnehmender Korngröße des Kristallpulvers an Intensität zunehmen und dem Lösungsspektrum ähnlicher werden. Dabei wird jedoch ein Grenzzustand erreicht insofern, als weiteres (auch tagelanges) Zerreiben des Pulvers Form und Höhe der Kurven nicht mehr ändert. Die Verflachung des Spektrums mit wachsender Teilchengröße kann auch hier als zunehmender Beitrag der regulären Reflexion zur Gesamtreflexion gedeutet werden ${ }^{11}$, die intensivste, nicht mehr weiter veränderliche Kurve IV würde dann einer praktisch vollkommen diffusen Reflexion entsprechen.

Wie diese Messungen zeigen, lassen sich bei genügend kleinen Einfalls- und Austrittswinkeln der Strahlung $\left([\alpha+\vartheta]<45^{\circ}\right)$ und bei genügend fein pulverisierten Stoffen reproduzierbare und quantitative Absorptionsspektren in Reflexion aufnehmen, die für den untersuchten Stoff in gleicher

10 Vorläufige Messungen an Pikraten zeigten ein ähnliches Ergebnis.
Weise charakteristisch sind wie die in Durchsicht gewonnenen Spektren.

Von besonderem Interesse ist die Beobachtung, daß die Lage des Maximums der Hauptbande von der Korngröße unabhängig ist, daß jedoch das längstwellige Maximum sich mit abnehmender Korngröße stark nach kurzen Wellen verschiebt und schließlich fast vollkommen mit der Hauptbande verschmilzt, wie es ja auch in wäßriger Lösung nur noch als geringe Inflexion in der abfallenden Kurve zu erkennen ist. Nach den Messungen von $\mathrm{Telto} \mathrm{w}^{9}$ bei $20^{\circ} \mathrm{K}$ handelt es sich bei diesem System um diffuse, teilweise von koniinuierlicher Absorption überdeckte Schwingungsbanden uneinheitlichen Abstands, die sich der Elektronenanregung überlagern und bei Raumtemperatur zusammenfließen. Sie werden als überlagerte Gitter- und Radikalschwingungen gedeutet. Diese Erklärung wird durch unsere Messungen stark gestuitzt, denn wenn bei dieser Elektronenanregung auch Schwingungsenergie an das Gitter abgegeben wird, muß die Potentialkurve des angeregten Zustands von den das angeregte Ion umgebenden Kraftfeldern abhängig werden, so dafs die Intensitätsverteilung und damit auch das Absorptionsmaximum sich mit zunehmender Vergrößerung der Oberfläche des Kristallpulvers verschieben muß.

11 Bei größeren Teilchen kann durch das Pressen des Pulvers eine bevorzugt ebene Lagerung der Kriställchen in der Oberfläche hervorgerufen werden, was stärkere reguläre Reflexion zur Folge haben muß.

\title{
Über den Energiemechanismus der Aminobenzoesäuren
}

\author{
Von Hermann Schüler und Adalbert Woeldike \\ Aus dem Kaiser-Wilhelm-Institut für Physik, Max-Planck-Institut, Hechingen \\ (Z. Naturforschg. 2a, 657-661 [1947]; eingegangen am 20. August 1947)
}

Es werden Befunde an 0 -, $m$ - und $p$-Aminobenzoesäure in Emission, in Absorption in gasförmiger Phase und in äthylalkoholischer Lösung diskutiert. Dabei zeigt sich, daß die niedrigste Anregung der C:O-Bindung, die im COOH-Substituenten enthalten ist, im Gebiet 3000 bis $4000 \AA$ sehr verschieden stark auftreten kann. Der physikalische Grund dieser Erscheinung liegt in der starken Änderung der Übergangswahrscheinlichkeit, und zwar ruft die Einführung eines $\mathrm{OH}$ - bzw. $\mathrm{NH}_{2}$-Substituenten in 0 -Stellung eine starke Übergangswahrscheinlichkeit hervor, die in $m$ - und noch viel mehr in $p$ Stellung abnimmt und bei der Benzoesäure, die keinen zweiten Substituenten enthält, nur als sehr schwach nachgewiesen werden kann.

Aus den Eigenschaften der $m$-Aminobenzoesäure ist zu entnehmen, daß nicht allein die räumliche Nähe der beiden Substituenten entscheidend sein kann, sondern die Anwesenheit des Benzolringes, dessen Glieder die Substituenten miteinander innermolekular verbinden, mitbestimmend ist.

Die Befunde an der $p$-Aminobenzoesäure in Lösung weisen darauf hin, daß hier - im Gegensatz zur $o$ - und $m$-Konfiguration - auch Doppelmoleküle gebildet werden. 